\title{
Ways of single-phase rectifiers energy efficiency improvement
}

\author{
Vladlen Ivanov \\ Novosibirsk State Technical University \\ Novosibirsk, Russia \\ NSTU \\ ivanov.etk@yandex.ru
}

\author{
Sergei Myatez \\ Novosibirsk State Technical University \\ Novosibirsk, Russia \\ NSTU \\ serg_y_7578@mail.ru \\ Andrei Kapustin \\ Novosibirsk State Technical University \\ Novosibirsk, Russia \\ NSTU \\ kapusta_nsk@mail.ru
}

\author{
Irina Alekseeva \\ Novosibirsk State Technical University \\ Novosibirsk, Russia \\ NSTU \\ alekseeva1201@mail.ru
}

\begin{abstract}
This paper introduces prospects for further development of a single zone-phase rectifier, aimed to research and improve it. A new radial type circuit and its advantages are provided as well. Based on these studies, the ways of single-phase rectifiers power factor improvement are suggested. The ladder four-phase rectifier is offered as a technical means which is based on a pulse-width rectified voltage control method. Efficiency of the pulse-width control method for rectifier with a ladder structure is evaluated. Ladder structure converters decrease losses in power semiconductors to $25 \%$; therefore, the future progress of the zone-phase control method will be useful for practical application.
\end{abstract}

Keywords - Four-zonal converter, sector regulation, power indicators, power factor, distortion coefficient, efficiency.

\section{INTRODUCTION}

The rotation speed control of DC traction motors is achieved by changing the current and voltage value. It becomes possible through the use of amplitude regulation. This type of regulation is based on the principle of changing secondary winding commutation combinations. The main disadvantage of amplitude regulation is impetuous steps of the output voltage value which cause the scorching of contacts. Intention to provide a smooth voltage regulation led to creation of the phase regulation method. This method is based on changing of thyristor opening delay. In this method, the average rectified value depends on opening delay duration. But the major disadvantage of this principle is that the great reactive power occurs by this method, because it generally reduces the energy indicators of the rectifier. All stated minuses are mostly eliminated in so called zone or zone-phase regulators. This type of regulation is a combination of amplitude and phase regulation. There are different options of zone-phase voltage regulation. They differ by the number of zones and voltage ratios. Nowadays four-zone regulators, built on thyristor bridge rectifying circuit, are getting widespread use.

One of the main indicators of single-phase controlled rectifier is the power factor that determines the energy efficiency of using electrical energy (in case of alternating current). The power factor value of widely used controlled rectifiers lays in the range from 0.2 to 0.85 .

Increasing the energy indicators of single-phase controlled rectifiers is possible in three ways:

- using new circuit design and control methods of the converter;

- using additional power equipment or assemblies of artificial commutation, such as a reactive power compensator, filters, correctors, etc.;

- organizational measures for the rational construction of power supply circuits [5].

A rational way to improve the energy performance of controlled rectifiers from the investment point of view is to 
use the new control methods for the inverter and to change all thyrisrors on fully controlled semiconductor devices.

The one-phase zone bridge rectifier of $\mathrm{AC}$ in contrast to others has a gradual acceleration in a traction mode, but also has not an optimal efficiency factors, e.g. power factor. In a normal electric train operation mode, its power factor does not exceed the value of 0.8 [3].

This phenomenon is explained by the forced delay, used in the four-phase converter with the bridge rectifying structure and two commutation circuits (small and big). Two circuits cause movement of the rectified voltage electrical center along the current in the input and output circuits.

The low value of power factor shows that there is high reactive power consumption and supply current forms distortion. In this case, the electric-traction network and train electrical equipment is loaded by the particular reactive current which causes the increased energy consumption in the traction mode.

\section{METHODS OF THE POWER FACTORS AND ZONE CONVERTER EFFICIENCY INCREASING}

The factors, which have influence on power factor, should be evaluated for the problem of increasing the power factor in the first rectifying zone:

$$
\chi=P / \sqrt{ }\left(P^{2}+Q^{2}+T^{2}\right)=K_{s} \cdot K_{d},
$$

where $P, Q$ and $T$ - active power, reactive power and the power of distortion, correspondingly; $K_{s}-$ shift coefficient, $K_{d}$ - distortion coefficient.

The circuit design development of the single-phase alternating current rectifier with high power indicators is complicated by the fact that the single-phase alternating current system is always unbalanced, compared, for example, to a three-phase system. Due to this, theoretically, taking into consideration generally accepted assumptions of static converter elements ideality [4], the formal analysis shows that the three-phase rectifier enables to provide the input power factor for inductive nature of load, approximately:

$$
\chi=3 / \pi \approx 0.955 \text {, }
$$

while the single-phase alternating current rectifier provides:

$$
\chi=(2 \cdot \sqrt{ } 2) / \pi \approx 0.9
$$

In this context, value $\chi$ of operated rectifier appears to be much dependent on the value of artificial rectifier semiconductors opening delay.

Zone-phase rectifiers have their own disadvantages (great quantity of PSD cascade connection in the chain of rectified current, sneak circuits of overlapping (commutation) and etc.), which do not allow values to reach their own theoretical maximum [6]. In practice, these performances decrease indicators by $10-15 \%$.
Commutation circuit nesting results in general commutation duration, which makes $\gamma_{01}+\gamma_{02}$. It not only visibly distorts the curve of rectified voltage but also restricts adjustment range $\alpha_{\mathrm{p}}$ on low bound from value $\alpha_{\mathrm{p} \text { min }}$ and up to value $\alpha_{\mathrm{p} \max }$ on upper bound (Figure 1).

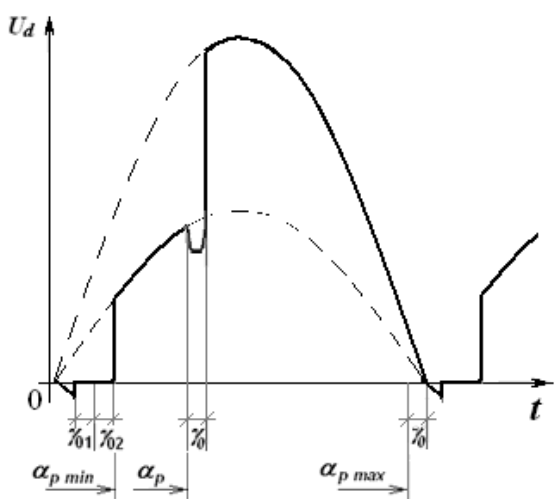

Fig.1. The influence of commutation angles on rectified voltage and the adjustment range of the zone-phase rectifier

The problem of double commutation circuit was eliminated in the improved version of the four-zone rectifier with a ladder structure (Fig. 2) and a new algorithm of thyristor control [2].

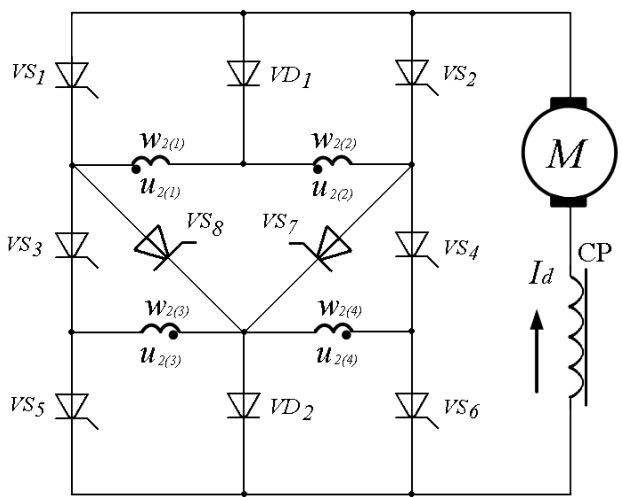

Fig.2. The power part of the four-zone rectifier with the ladder structure scheme

Owing to a special circuit design topology, the general commutation process duration is reduced by half for the majority of operation zones. This allows increasing the power factor by $4-5 \%$. In addition, the decreasing of switching angle $\gamma$ decreases capacity and dimensions of the static reactive power compensator equivalently, in case of their application for AC electric locomotives.

One of the ways to solve the problem of $\mathrm{AC}$ electric freight locomotives energy efficiency upgrading is the zone rectifier`s control algorithm improvement [3].

As a technical mean of increasing the energy efficiency, the improved four-zone rectifier with a so called semiconductor distortion factor switch ladder structure [1] was proposed by the authors.

The increasing of the $\mathrm{AC}$ freight electric train energy effectiveness by the zone rectifier's control algorithm improvement is the effective solution [2]. 
As the creation of the rectifier opening delay in the zone rectifier could not be refused, the distortion compensation fundamental component should be made for the power coefficient value, increasing in the first zone.

This principle is known as a sector control method. For common SCR-semiconductor switches, forced commutation is attended with the commutation key out circuits' injection which is not cost-effective. However, power semiconductors perfection allows using the sector control method with a new element base.

In that case, smooth regulation of output voltage will be provided by simultaneous shift of leading $(\alpha)$ and back $(\beta)$ edges where phase shift $(\varphi)$ of grid current main harmonic does not occur (Fig. 3).

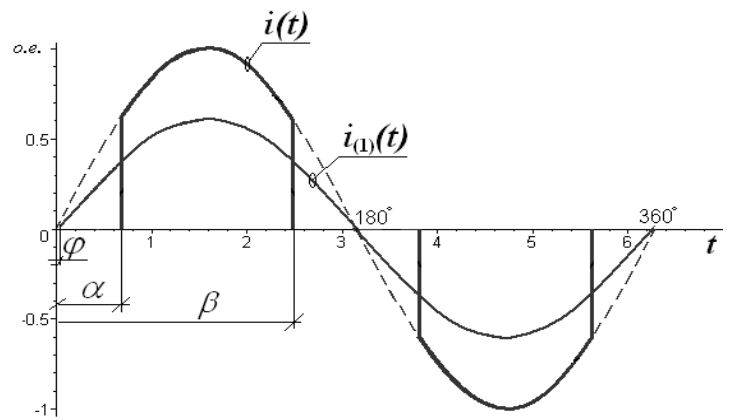

Fig.3. Grid current curve and fist harmonic of rectifier output current in case of sector regulation

The sector control method increases the transformation energy efficiency, so $K_{s} \leq 1,0$. The sector control method effect is carefully described in source [3].

For increasing the additional power factor, it is necessary to increase the distortion factor value. The distortion factor is the ratio of the first current harmonic effective range of primary winding to the effective range of the primary current.

To reduce the influence of the higher harmonic component on the power factor, the usage of controlling pulse-width for the rectifier first zone voltage was offered.

The pulse-width control method is a method of the average on-load voltage value by means of the pulse duty factor and the control key.

The pulse-width control method is realized as the sector control method - with the help of the semiconductors' switches substituted with fully controlled power semiconductors.

Efficiency is defined by the ratio of output transformer's active power to active power in its input. Applying to rectifying performance of the zone-phase converter means [4]:

$$
\eta=P_{d} / P_{I}=P_{d} /\left(P_{d}+\Delta P_{\text {lost }}\right)
$$

where $\Delta P_{\text {lost }}$ - losses of active power in the zone-phase converter.

These losses are the sum of the losses in the transformer and losses in rectifier elements - thyristors and diodes:

$$
\Delta P_{\text {lost }}=\Delta P_{t r}+\Delta P_{t h}+\Delta P_{d i o}
$$

According to these arguments, it is possible to say that efficiency of the converter increases through decreasing the sum of thyristor and diode losses $\Delta P_{t r}+\Delta P_{d i o}$. In other words, efficiency increases owing to a decreasing number of thyristors and diodes through which current passes at the same time.

Let us compare the equivalent circuits of bridge and ladder converters (Fig.4 and Fig.5) in their second zone of regulation. Four power semiconductors are involved in the bridge converter, and three power semiconductors are involved in the ladder converter.

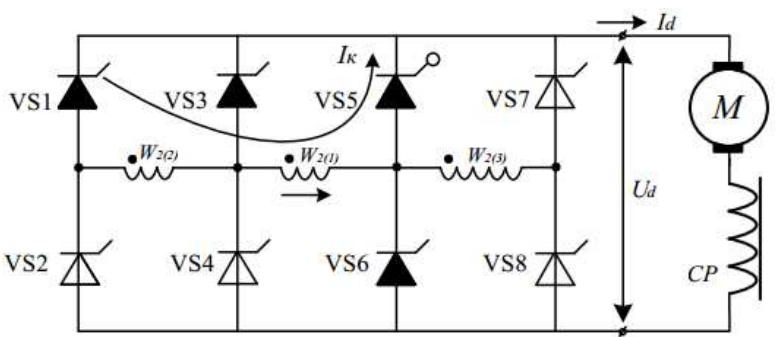

Fig.4. The equivalent circuit of the bridge rectifier converter for the second regulation zone

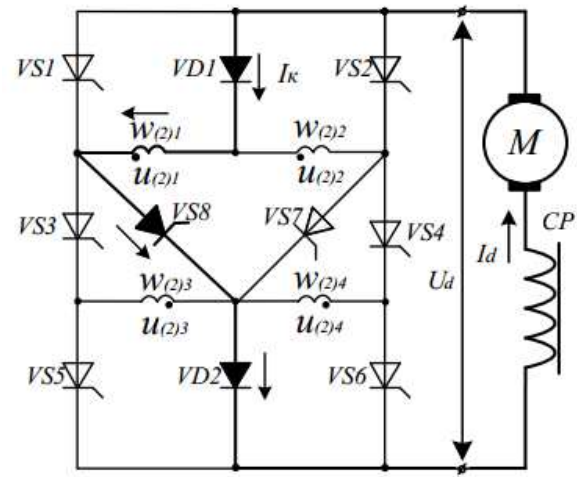

Fig.5. The equivalent circuit of the ladder rectifier converter for the second regulation zone

Thus one can increase the efficiency of the converter by decreasing power semiconductor losses by $25 \%$.

The further improvement of circuit topology with zonephase control will provide a decrease of the number of power semiconductors both in the circuit as a whole and on the way of current in each zone. In this case, circuit will have a higher power factor and less commutation losses. Taking into account these advantages, the zone-phase circuit is expedient to use in practice.

\section{ANALYTICAL MODELING}

In analytical modeling environment "MathCad", the rectifier model was made by the authors to make the current curves' harmonic analyses in the presence of the pulse-width voltage. For the analytical model with pulse-width controlling, unipolar signal front sweep was used.

PWM modulation is performed by applying voltage control of the control system (modulating voltage) of a certain 
form to the input. This voltage is compared with a saw tooth reference voltage (deploy). When the amplitude of the modulating voltage is changed, the magnitude of the output voltage changes [8].

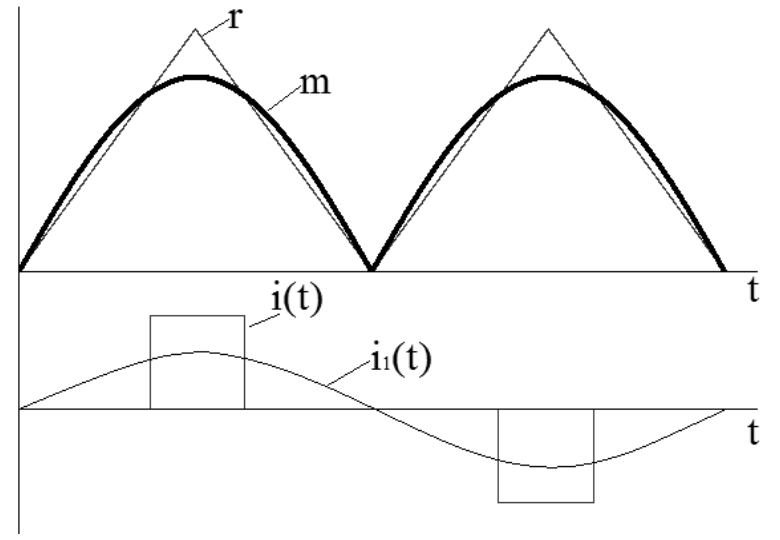

Fig.6. The rectifier model with the sector control in the first controlling zone

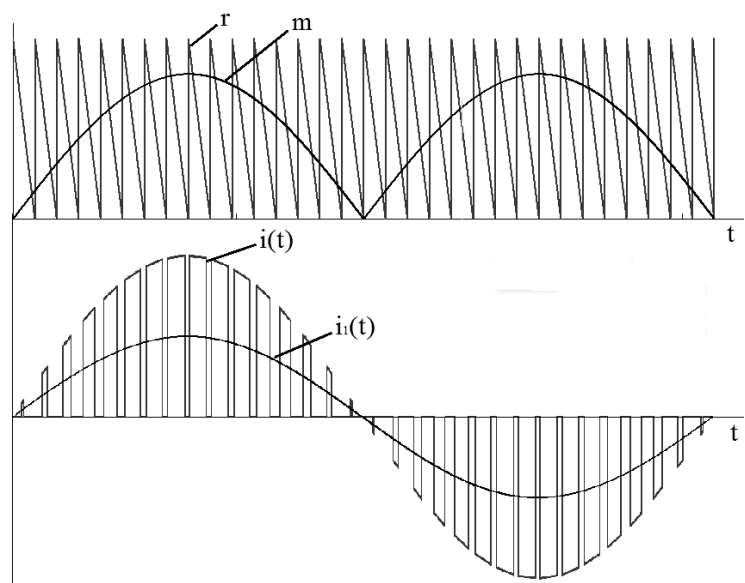

Fig.7. The rectifier model with the pulse-width control in the first controlling zone

With help of this model, the output current spectrum of the rectifier was defined (Fig.8 and 9).

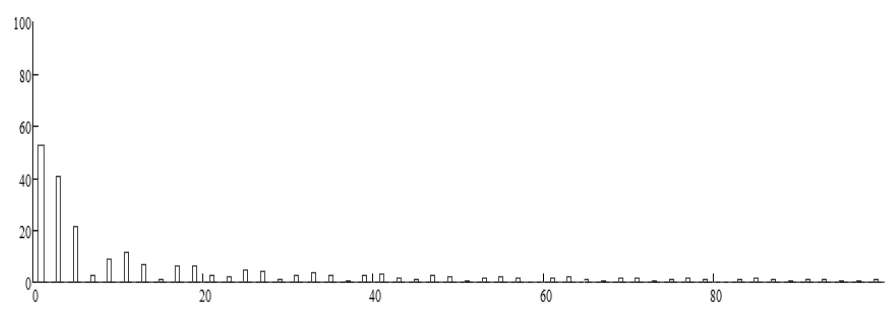

Fig.8. The output current spectrum of the sector controlling rectifier

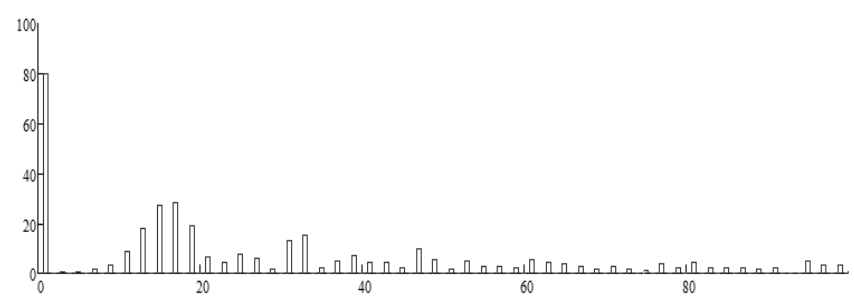

Fig.9. The output current spectrum of the pulse-width controlling rectifier
The result of the harmonic analysis shows dependence of distortion on the fill factor. The comparison of the phase, sector and pulse-width voltage regulation methods are presented in figure 10. The graph shows that the principles of the sector and pulse-width controlling methods are significantly more effective than those of the phase control method. Values of curve 2 (Fig.10) are above curve 1 on average by $22 \%$, but the usage of the pulse-width controlling method increases the power factor within the first $10 \%$ of the regulation range, changing the duty factor (D) from 0 to 1 .

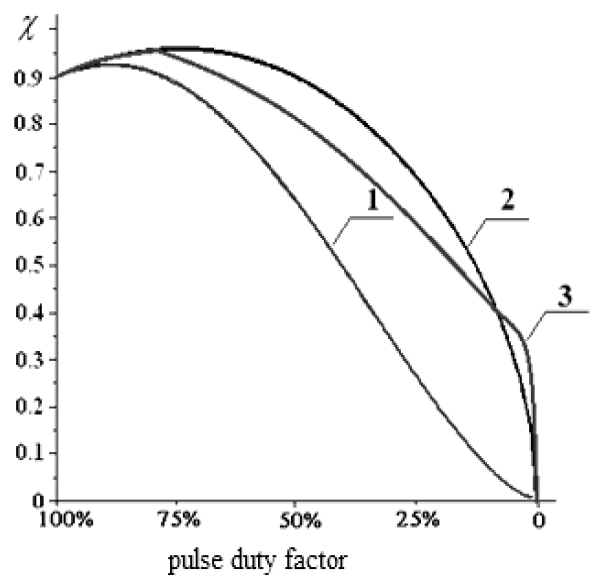

Fig.10. The differences between the power factors of the ladder structure rectifier 1 - phase controlling 2 - sector controlling 3 - pulse-width controlling on the first zone

The result of the pulse width modulation principle for the rectifier shows that the power factor of pulse width modulation surpasses the power factor of the sector method only within the first $8.2 \%$ of the control range.

\section{CONCLUSIONS}

The results' analysis of zone-phase regulator circuit shows that the ways of improving energy performance are oriented towards the development and improvement of new circuit design and control principles.

The further improvement of these zone-phase control circuits makes it possible to decrease the number of power semiconductors, to decrease commutation losses, to increase power factor, and finally, to increase efficiency. Converters with ladder topology decrease losses in power semiconductors by $25 \%$; thus with regard to all pluses, the future progress of zone-phase control circuits is useful for practical application.

Rectifiers with the ladder structure have a less switching processes' period compared to that of the bridge rectifier. The application of the ladder structure rectifier as the element of the AC electric locomotives traction converters allows one to improve the energy performance and, first of all, the power factor by an average of $2-4 \%$. Theoretically, sector regulation increases the average power factor by $25-30 \%$.

As the analytical modeling shows, the means of the pulsewidth controlling principle increases the rectifier's energy indicators by $10 \%$ within the first controlling range. Authors offer to combine sector and pulse-width methods of controlling the voltage for obtaining better energy indicators. 


\section{References}

[1] V. V. Ivanov, S. V. Myatez, A. V. Kapustin and I. K. Alekseeva, "Development prospects of single-phase zone rectifiers", The 11 International forum on strategic technology (IFOST 2016), Novosibirsk : NSTU, Pt. 2, pp. 105-107, 2016.

[2] A.V. Plaks, Control systems of electric rolling stock, M.: Training Center for Education in rail transport, 2005, p. 360.

[3] G.S. Zinovev, Bases of the power electronics, Novosibirsk, NSTU, 2003, p. 664.

[4] V. V. Ivanov, S. V. Myatez, E. G. Langeman and N. I. Schurov, "Pulsewidth control in ladder structure four-phase rectifier for ACelectromotive", IOP Conf. Series: Materials Science and Engineering, 127(1), 012004, 2016.

[5] S. K. Kaper and N. K. Choudhary, "A review of power management and stability issues in microgrid", IEEE 1st International Conference on Power Electronics, Intelligent Control and Energy Systems (ICPEICES), 2016, 1, pp. 1-6.

[6] B.G. Yuzhakov, Electric drive and converters of rolling stock: Textbook for technical schools and colleges of rail transport, M.: SEI Educational Centre for Education in rail transport, 2007, p. 396.

[7] L.A. Bessonov, Theoretical Foundations of Electrical Engineering, Electrical circuits: Ucheb.dlya university students, M .: Gardariki, 1987. p. 293.

[8] N. W. Lidula and A. D. Rajapakse, "Microgrids research: a review of experimental microgrids and test systems", Renewable and Sustainable Energy Reviews, 15, pp. 186-202, 2011.

[9] E. Y. Abramov, A. A. Stang and S. A. Enkudinov, "Transformation of the urban electric transport system when using autonomous energy sources", Advanced Materials Research High technology: research and applications, 1040, pp. 714-718, 2014.

[10] S.A. Evdokimov and L.G. Evdokimova, One-phase AC-DC rectifier, Patent of Russia №2398344, 2009.

[11] B. Kroposki, R. Lasseter, T. Ise, S. Morozumi, S. Papathanassiou and N. Hatziargyriou, "A look at microgrid technologies and testing projects from around the world, making microgrids work", IEEE Power and EnergyMagazine, 6, pp. 40-53, 2008.

[12] B. A. Arzhannikov and A. A. Pyshkin, Improving of DC power supply system based on automatic voltage regulation of traction substations, Ekaterinburg: USURT Press, 2006, p. 116.

[13] E. Y. Abramov, "Experimental investigation of energy parameters of urban electric transport traction substations", Proceedings of the Russian higher school academy of science, 3 (32), pp. 33-42, 2016.

[14] A. A. Stang, E. A. Spiridonov and M. V. Yaroslatsev, "Application of energy storage devices in power systems of urban electric transport" Transport of the Russian Federation, 3, pp. 68-70, 2012.

[15] V. V. Biryukov and A. V. Kulekina, "The calculation features of the electrical energy storage devices parameters in transport", The 11 International forum on strategic technology (IFOST 2016), 2, pp. 41-43, 2016.

[16] V. E. Rozenfeld, "Analytical calculation of electric railway networks The theoretical and scientific-practical peer-reviewed journal", Elektrichestvo, 9, pp. 6-17, 1947. 